\title{
ERRATA
}

\section{Erratum: Nonlinear low-temperature magnetization of the quasi-one-dimensional charge-density-wave conductor $\mathrm{K}_{0.3} \mathrm{MoO}_{3}$ [JETP Lett. 68, No. 4, 301-306 (25 August 1998)]}

D. V. Eremenko and A. V. Kuznetsov

Moscow Engineering-Physics Institute, 115409 Moscow, Russia

S. V. Zaĭtsev-Zotov

Institute of Radio Engineering and Electronics, Russian Academy of Sciences, 103907 Moscow, Russia

V. N. Trofimov

Joint Institute of Nuclear Research, 141980 Dubna, Moscow Region, Russia

Pis'ma Zh. Éksp. Teor. Fiz. 68, 852 (10 December 1998)

[S0021-3640(98)01423-6]

PACS numbers: 99.10.+g, 75.45.+j, 71.45.Lr, 75.60.Ej

The following sentence should be appended to the caption of Fig. 2 on page 303:

The squares and the dotted curve show the magnetization of the sample at $T=254.2 \mathrm{~K}$. 